\title{
THE COMPLEXITY OF CONTINUOUS EMBEDDABILITY BETWEEN DENDRITES
}

\author{
ALBERTO MARCONE AND CHRISTIAN ROSENDAL
}

\begin{abstract}
We show that the quasi-order of continuous embeddability between finitely branching dendrites (a natural class of fairly simple compacta) is $\Sigma_{1}^{1}$-complete. We also show that embeddability between countable linear orders with infinitely many colors is $\boldsymbol{\Sigma}_{1}^{1}$-complete.
\end{abstract}

§1. Introduction. In [LR02] Louveau and Rosendal initiated the study of the complexity of $\Sigma_{1}^{1}$ (i.e., analytic) quasi-orders on Polish (i.e., separable and completely metrizable) spaces. This study yields results about the complexity of the equivalence relation induced by the quasi-order and thus contributes to the ongoing study of analytic equivalence relations. The equivalence relations obtained in this way are quite different from the ones induced by a Polish group action (the literature about the latter is extensive, see e.g., [BK96] and [Hjo00]).

Recall that a quasi-order is a reflexive and transitive binary relation (so that equivalence relations and partial orders are particular kinds of quasi-orders). The induced equivalence relation is obtained by declaring equivalent two elements if and only if each of them precedes the other in the quasi-order.

Definition 1.1. If $R$ and $S$ are quasi-orders defined on Polish spaces $X$ and $Y$ we say that $S$ is Borel reducible to $R$, and write $S \leq_{\mathrm{B}} R$, if there exists a Borel function $f: Y \rightarrow X$ such that

$$
\forall x, y \in Y(x S y \longleftrightarrow f(x) R f(y)) .
$$

A $\Sigma_{1}^{1}$ quasi-order $R$ is $\Sigma_{1}^{1}$-complete if $S \leq_{\mathrm{B}} R$ for any $\boldsymbol{\Sigma}_{1}^{1}$ quasi-order $S$.

If $R$ is $\Sigma_{1}^{1}$-complete it follows that the equivalence relation induced by $R$ is $\Sigma_{1}^{1}$ complete among equivalence relations and hence immensely more complicated than any equivalence relation induced by a Polish group action.

In [LR02] Louveau and Rosendal proved that several natural $\boldsymbol{\Sigma}_{1}^{1}$ quasi-orders are $\Sigma_{1}^{1}$-complete. Here we sharpen one of their results and, in doing so, we prove that another quasi-order of some independent interest is also $\Sigma_{1}^{1}$-complete (see Theorem 3.2).

Received May 25, 2003; revised January 16, 2004.

2000 Mathematics Subject Classification. Primary 03E15; Secondary 54F15.

This research was carried out during Fall 2002, while both authors were visiting members of the Fields Institute in Toronto during the "Thematic Program on Set Theory and Analysis". The authors thank the Fields Institute for its hospitality. The first author was partially supported by INdAM of Italy. 
Let $I=[0,1]$ so that $I^{2}$ is the unit square. Any space homeomorphic to $I$ is called an arc. The space $\mathrm{K}\left(I^{2}\right)$ of all compact subsets of $I^{2}$ equipped with the Vietoris topology is a Polish space (a complete metric is the Hausdorff metric). Let $\sqsubseteq^{c}$ be the $\Sigma_{1}^{1}$ quasi-order of continuous embeddability between compact metric spaces. Louveau and Rosendal proved that $\complement^{c}$ is $\Sigma_{1}^{1}$-complete on $\mathrm{K}\left(I^{2}\right)$ (and hence on $\mathrm{K}\left(I^{n}\right)$ for any $n$ with $\left.2 \leq n \leq \aleph_{0}\right)$.

Recall that a continuum is a compact and connected metric space. The space $\mathrm{C}\left(I^{2}\right)$ of all continua contained in $I^{2}$ is a closed subspace of $\mathrm{K}\left(I^{2}\right)$, and hence is itself Polish with respect to the Vietoris topology. Louveau and Rosendal's proof actually shows that $\sqsubseteq^{c}$ is $\Sigma_{1}^{1}$-complete on $\mathrm{C}\left(I^{2}\right)$. We are interested in further restrictions of $\sqsubseteq^{c}$.

Definition 1.2. A dendrite is a locally connected (also called Peano) continuum which contains no subcontinuum homeomorphic to the circle $S^{1}$.

Dendrites are an important class of continua, and the textbook [Nad92] devotes a whole chapter to their study. Every dendrite is homeomorphic to a subset of $I^{2}$ and dendrites are a $\boldsymbol{\Pi}_{3}^{0}$ (indeed $\boldsymbol{\Pi}_{3}^{0}$-complete) subset of $\mathrm{C}\left(I^{2}\right)$ (see [CDM02] for a proof of this and several other results about dendrites from the viewpoint of descriptive set theory). The equivalence relation of homeomorphism between dendrites is strictly simpler (in the sense of Borel reducibility) than the same equivalence relation between arbitrary continua. Indeed the former is classifiable by countable structures [CDM02, §6], while the latter is not [Hjo00, §4.3]. Therefore it is natural to ask whether mutual continuous embeddability is simpler on dendrites than on arbitrary continua. We answer this question in the negative by showing that even on a fairly small collection of dendrites $\sqsubseteq^{c}$ is still $\Sigma_{1}^{1}$-complete.

Definition 1.3. If $X$ is a continuum and $x \in X$ the order of $x$ in $X$, denoted by $\operatorname{ord}(x, X)$, is the smallest cardinal number $\kappa$ such that there exists a neighborhoodbase for $x$ in $X$ consisting of open sets each with boundary of cardinality less than or equal to $\kappa$.

A point $x \in X$ is a branching point of $X$ if $\operatorname{ord}(x, X)>2$.

A continuum $X$ is finitely branching if $\operatorname{ord}(x, X)$ is finite for every $x \in X$.

These notions provide the following presentation theorem for dendrites (see [Nad92, Corollary 10.28]): each nondegenerate dendrite $X$ can be written as $X^{[1]} \cup \bigcup_{n \in \mathbb{N}} A_{n}$, where $X^{[1]}=\{x \in X \mid \operatorname{ord}(x, X)=1\}$ (this set may be uncountable), each $A_{n}$ is homeomorphic to $I$, and $A_{n} \cap \bigcup_{m<n} A_{m}$ consists of a single point, which is one of the two end points of $A_{n}$.

The following lemma implies that the space of finitely branching dendrites is a standard Borel space, i.e., Borel isomorphic to a Polish space (see [Kec95] for details). It is clear that for the purpose of studying Borel reducibility we can consider standard Borel spaces rather than Polish spaces.

LEMma 1.4. The set of finitely branching dendrites is a Borel subset of $\mathrm{C}\left(I^{2}\right)$.

Proof. Let $\mathscr{D} \subset \mathrm{C}\left(I^{2}\right)$ be the (Borel) set of all dendrites. If $X \in \mathscr{D}$ there are only countably many $x \in X$ which are branching points of $X(\operatorname{see}[\mathrm{Nad} 92$, Theorem 10.23]). Moreover there exists Borel functions $b_{n}: \mathscr{D} \rightarrow I^{2}$ such that $\left\{x \in I^{2} \mid\right.$ $x$ is a branching point of $X\}=\left\{b_{n}(X) \mid n \in \mathbb{N}\right\}$ for all $X \in \mathscr{D}$ (see the proof of Lemma 6.5 in [CDM02]). 
Since $X \in \mathrm{C}\left(I^{2}\right)$ is a finitely branching dendrite if and only if $X \in \mathscr{D}$ and $\forall n \exists k \operatorname{ord}\left(b_{n}(X), X\right) \leq k$, it suffices to show that the set

$$
\left\{(X, x) \in \mathrm{C}\left(I^{2}\right) \times I^{2} \mid X \in \mathscr{D} \& \operatorname{ord}(x, X)>k\right\}
$$

is Borel for every $k \geq 2$. In [CDM02, Lemma 6.4] this is done for $k=2$, and a straightforward generalization of that proof yields the result for every $k$.

We can now state the main result of the paper.

MAIN THEOREM. The quasi-order $\sqsubseteq^{c}$ restricted to finitely branching dendrites is $\Sigma_{1}^{1}$-complete.

We now explain the organization of the paper. In section 2 we fix our notation and recall the results of [LR02] that we will use. In section 3 we give a combinatorial example of a $\boldsymbol{\Sigma}_{1}^{1}$-complete quasi-order. This example, which involves colorings of countable linear orders, is of independent interest and will be used in the proof of the Main Theorem together with the technical result proved in section 4. The latter deals with a special kind of order preserving maps from $\mathbb{Q}$ into itself. In section 5 we complete the proof of the Main Theorem.

§2. Notation and previous results. We use $\mathbb{N}<\mathbb{N}$ for the sets of all finite sequences of natural numbers; $2^{<\mathbb{N}} \subset \mathbb{N}^{<\mathbb{N}}$ consists of the sequences mentioning only 0 and 1. $\mathbb{N}^{\mathbb{N}}$ and $2^{\mathbb{N}}$ are the corresponding sets of infinite sequences. If $s \in \mathbb{N}<\mathbb{N},|s|$ is its length and when $i<|s|, s(i)$ is the $(i+1)$-th element of $s$; if $n \leq|s|, s\lceil n$ is the initial segment of $s$ of length $n$. The same notations apply also to infinite sequences. If $s \in \mathbb{N}<\mathbb{N}$ and $k \in \mathbb{N}, s \neg k$ is the sequence obtained by adding $k$ at the end of $s . \emptyset$ is the unique sequence of length 0 . The relation of being an initial segment between sequences is denoted by $\subset$. We use $\leq_{\text {lex }}$ to denote lexicographic order on any sets of sequences whose elements have a natural order, and in particular on $\mathbb{N}<\mathbb{N}$. In particular $s \subset t$ implies $s<$ lex $t$.

Definition 2.1. If $s, t \in \mathbb{N}<\mathbb{N}$ we say that $s$ is pointwise dominated by $t$, and write $s \leq_{\mathrm{pw}} t$, to mean that $|s|=|t|$ and $s(i) \leq t(i)$ for every $i<|s|$.

Definition 2.2. A function $f: \mathbb{N}^{<\mathbb{N}} \rightarrow \mathbb{N}^{<\mathbb{N}}$ is Lipschitz if it preserves both extension and length, i.e., $s \subset t \longrightarrow f(s) \subset f(t)$ and $|f(s)|=|s|$.

Definition 2.3. A tree on $2 \times \mathbb{N}$ is a subset $T$ of $2^{<\mathbb{N}} \times \mathbb{N}<\mathbb{N}$ such that $(u, s) \in T$ implies $|u|=|s|$ and $(u\lceil n, s \mid n) \in T$ for every $n<|s|$.

If $T$ is such a tree and $s \in \mathbb{N}^{<\mathbb{N}}$ we let $T(s)=\left\{u \in 2^{<\mathbb{N}} \mid(u, s) \in T\right\}$.

Definition 2.4. A tree $T$ on $2 \times \mathbb{N}$ is normal if $\forall s \in \mathbb{N}<\mathbb{N} T(s) \neq \emptyset$ and $T(s) \subseteq$ $T(t)$ whenever $s \leq_{\text {pw }} t$. Let $\mathscr{T}$ be the set of all normal trees on $2 \times \mathbb{N}$.

$\mathscr{T}$ is a closed subset of $2^{2^{<N} \times \mathbb{N}^{<N}}$ and hence a Polish space.

Definition 2.5. If $T, S \in \mathscr{T}$ let

$$
\begin{aligned}
T \leq_{\max } S & \Longleftrightarrow \exists f: \mathbb{N}^{<\mathbb{N}} \rightarrow \mathbb{N}^{<\mathbb{N}} \text { Lipschitz } \forall s \in \mathbb{N}^{<\mathbb{N}} T(s) \subseteq S(f(s)) ; \\
T \mathscr{R} S & \exists \alpha, \beta \in \mathbb{N}^{\mathbb{N}} \forall n T(\alpha \mid n) \subseteq S(\beta\lceil n) .
\end{aligned}
$$

It is straightforward that $T \leq_{\max } S$ implies $T \mathscr{R} S$. Notice that $\leq_{\max }$ is a quasi-order, while $\mathscr{R}$ lacks transitivity and is only a binary relation. 
In our discussion of Borel reducibility it will be useful to use the following extension of the original notion.

Definition 2.6. Let $E \subseteq F$ and $R \subseteq S$ be $\Sigma_{1}^{1}$ binary relations on Polish spaces $X$ and $Y$ respectively. We say that $(E, F)$ is Borel reducible to $(R, S)$, and write $(E, F) \leq_{\mathrm{B}}(R, S)$, if and only if there exists a Borel function $f: X \rightarrow Y$ such that $x E y \longrightarrow f(x) R f(y)$ and $\neg x F y \longrightarrow \neg f(x) S f(y)$.

One sees easily that $\leq_{\mathrm{B}}$ is a quasi-order and that, if we write simply $E$ in place of $(E, F)$ when $E=F$, it extends the notion of Borel reducibility defined at the beginning of the paper.

In [LR02, Theorem 2.5] Louveau and Rosendal proved that $\leq_{\max }$ is $\Sigma_{1}^{1}$-complete, but - as they noticed - their proof actually gives sharper results. The one we will use is the following.

Theorem 2.7. Any $\Sigma_{1}^{1}$ quasi-order $S$ defined on a Polish space $X$ is Borel reducible to $\left(\leq_{\max }, \mathscr{R}\right)$, i.e., there exists a Borel function $f: X \rightarrow \mathscr{T}$ such that for any $x, y \in X$

(1) if $x$ Sy then $f(x) \leq_{\max } f(y)$;

(2) if $f(x) \mathscr{R} f(y)$ then $x S y$.

We will need the following fact about $\leq_{\max }$.

Lemma 2.8. If $T, S \in \mathscr{T}$ are such that $T \leq_{\max } S$ then there exists $g: \mathbb{N}<\mathbb{N} \rightarrow \mathbb{N}<\mathbb{N}$ which is both Lipschitz and $\leq$ lex -preserving such that $\forall s \in \mathbb{N}<\mathbb{N} T(s) \subseteq S(g(s))$.

Proof. Suppose $f: \mathbb{N}^{<\mathbb{N}} \rightarrow \mathbb{N}^{<\mathbb{N}}$ is a Lipschitz function witnessing $T \leq_{\max } S$. Let $f^{*}: \mathbb{N}^{<\mathbb{N}} \times \mathbb{N} \rightarrow \mathbb{N}$ be such that $f\left(s^{\frown} n\right)=f(s)^{\frown} f^{*}(s, n)$ for every $s \in \mathbb{N}^{<\mathbb{N}}$ and $n \in \mathbb{N}$.

Define $g^{*}: \mathbb{N}^{<\mathbb{N}} \times \mathbb{N} \rightarrow \mathbb{N}$ by

$$
g^{*}(s, n)=\max \left\{\left\{f^{*}(s, m)+1 \mid m<n\right\}, f^{*}(s, n)\right\},
$$

and let $g$ be defined by $g(\emptyset)=\emptyset, g\left(s^{\wedge} n\right)=g(s) \wedge g^{*}(s, n)$.

It is immediate that $g$ preserves $\leq_{\text {lex }}$ and by induction it is straightforward to show that $f(s) \leq_{\mathrm{pw}} g(s)$ for every $s \in \mathbb{N}<\mathbb{N}$. Since $S$ is normal and $f$ is Lipschitz, this implies that $g$ is also a witness to $T \leq{ }_{\max } S$.

§3. Coloring linear orders with infinitely many colors. Let LO be the set of all strict linear orders with domain $\mathbb{N}$. LO can be viewed as a closed subset of $2^{\mathbb{N}^{2}}$, and hence it is a Polish space.

Definition 3.1. Let $\mathbb{N}^{\mathrm{LO}}=\mathrm{LO} \times \mathbb{N}^{\mathbb{N}}$. If $\mathfrak{A}=\left(L_{A}, f_{A}\right)$ and $\mathfrak{B}=\left(L_{B}, f_{B}\right)$ are two elements of $\mathbb{N}^{\mathrm{LO}}$ we define $\mathfrak{A} \leq_{\mathbb{N} L O} \mathfrak{B}$ if and only if there exists $\psi: \mathbb{N} \rightarrow \mathbb{N}$ such that

(i) $a L_{A} a^{\prime}$ implies $\psi(a) L_{B} \psi\left(a^{\prime}\right)$ for every $a, a^{\prime} \in \mathbb{N}$;

(ii) $f_{A}(a)=f_{B}(\psi(a))$ for every $a \in \mathbb{N}$.

An element of $\mathbb{N}^{\mathrm{LO}}$ can be viewed as a countable linear order whose elements are colored with infinitely many colors. One such colored linear order is $\leq_{\mathbb{N} L O}$ another if there is an order-and-color-preserving map from the former into the latter. $\leq_{\mathbb{N} L O}$ is clearly a $\boldsymbol{\Sigma}_{1}^{1}$ quasi-order on the Polish space $\mathbb{N}^{\mathrm{LO}}$.

After we proved the following Theorem we learned that Louveau previously obtained the same result by different means. 
THEOREM 3.2. The quasi-order $\leq_{\mathbb{N}^{L O}}$ on $\mathbb{N}^{\mathrm{LO}}$ is $\boldsymbol{\Sigma}_{1}^{1}$-complete.

Proof. By Theorem 2.7 it suffices to show that $\left(\leq_{\max }, \mathscr{R}\right) \leq_{\mathrm{B}} \leq_{\mathbb{N}^{L O}}$. To this end we define $\mathfrak{A}_{T}=\left(L_{T}, f_{T}\right) \in \mathbb{N}^{\mathrm{LO}}$ for every $T \in \mathscr{T}$. For notational convenience we think of $L_{T}$ as a linear order on $T$ (rather than $\mathbb{N}$ ) and of $f_{T}$ as a function with domain $T$ and range the countable set $2^{<\mathbb{N}}$. It is easy to transform such an object into a full-fledged element of $\mathbb{N}^{\mathrm{LO}}$. Let

$$
(u, s) L_{T}(v, t) \Longleftrightarrow s<_{\operatorname{lex}} t \vee\left(s=t \& u<_{\operatorname{lex}} v\right)
$$

and $f_{T}(u, s)=u$.

The function $\mathscr{T} \rightarrow \mathbb{N}^{\mathrm{LO}}, T \mapsto \mathfrak{A}_{T}$, obtained by combining this definition with the transformation hinted above, is continuous. To complete the proof we need to show:

(1) if $T \leq_{\max } S$ then $\mathfrak{A}_{T} \leq_{\mathbb{N}^{L O}} \mathfrak{A}_{S}$;

(2) if $\mathfrak{A}_{T} \leq_{\mathbb{N}^{L O}} \mathfrak{A}_{S}$ then $T \mathscr{R} S$.

(1) Suppose $T \leq_{\max } S$ and, by Lemma 2.8 , let $f: \mathbb{N}<\mathbb{N} \rightarrow \mathbb{N}<\mathbb{N}$ be a Lipschitz and $\leq_{\text {lex }}$-preserving function such that $\forall s \in \mathbb{N}<\mathbb{N} T(s) \subseteq S(f(s))$. Let $\psi: T \rightarrow S$ be defined by $\psi(u, s)=(u, f(s))$. It is immediate that $\psi$ witnesses $\mathfrak{A}_{T} \leq_{\mathbb{N} L O} \mathfrak{A}_{S}$.

(2) Suppose $\psi$ witnesses $\mathfrak{A}_{T} \leq_{\mathbb{N} L O} \mathfrak{A}_{S}$. Since $u=f_{T}(u, s)=f_{S}(\psi(u, s))$ for every $(u, s) \in T$, we have $\psi(u, s)=(u, \varphi(u, s))$ for some function $\varphi: T \rightarrow \mathbb{N}<\mathbb{N}$ which is length preserving and such that $\varphi(u, s) \leq_{\operatorname{lex}} \varphi(v, t)$ whenever $(u, s),(v, t) \in$ $T$ are such that $(u, s) L_{T}(v, t)$.

We define inductively $\alpha, \beta \in \mathbb{N}^{\mathbb{N}}$ so that at stage $n$ we have already defined $\alpha\lceil(n+1)$ and $\beta\lceil n$ satisfying the following conditions:

(a) $\varphi(u, t) \supset \beta\lceil n$ for every $t \supset \alpha\lceil(n+1)$ and $u \in T(t)$;

(b) for some $v \in T\left(\left(\alpha\lceil n)^{\frown}(\alpha(n)+1)\right)\right.$ we have $\varphi\left(v,\left(\alpha\lceil n)^{\frown}(\alpha(n)+1)\right) \supset \beta\lceil n\right.$;

(c) $T(\alpha\lceil n) \subseteq S(\beta\lceil n)$.

Obviously this suffices to show that $T \mathscr{R} S$.

We start with $\alpha(0)=0$ and notice that (a)-(c) are trivially satisfied $(T(\emptyset)=\{\emptyset\}$ for any $T \in \mathscr{T})$.

Now suppose $\alpha \uparrow(n+1)$ and $\beta\lceil n$ have been defined and satisfy (a)-(c): we need to define $\alpha(n+1)$ and $\beta(n)$. By (b) let $v \in T\left(\left(\alpha\lceil n)^{\frown}(\alpha(n)+1)\right)\right.$ and $j \in \mathbb{N}$ be such that $\varphi\left(v,(\alpha\lceil n) \frown(\alpha(n)+1))=\left(\beta\lceil n)^{\frown} j\right.\right.$.

By the properties of $\varphi$ and by (a), for every $k \in \mathbb{N}$ and $u \in T((\alpha \uparrow(n+1)) \frown k)$ we have $\beta\left\lceil n \subset \varphi\left(u,\left(\alpha\lceil(n+1))^{\frown} k\right) \leq_{\text {lex }}\left(\beta\lceil n)^{\frown} j\right.\right.\right.$ and hence $\varphi\left(u,\left(\alpha\lceil(n+1))^{\frown} k\right)(n) \leq\right.$ $j$. Using again the properties of $\varphi$, this implies that for some $k$ and $j^{\prime} \leq j$ we have $\varphi\left(u,\left(\alpha\lceil(n+1))^{\frown} k\right)(n)=\varphi\left(u,(\alpha \uparrow(n+1))^{\frown}(k+1)\right)(n)=j^{\prime}\right.$ for every $u \in T\left(\left(\alpha\lceil(n+1))^{\wedge} k\right)\right.$. Let $\alpha(n+1)=k$ and $\beta(n)=j^{\prime}$ for such $k$ and $j^{\prime}$.

(a) and (b) follow immediately from the properties of $\varphi$. To prove (c) fix $v \in$ $T(\alpha \uparrow(n+1))$ and let $u \in T(\alpha \uparrow(n+2))$ be arbitrary (recall that $T(s) \neq \emptyset$ for every $s \in \mathbb{N}<\mathbb{N}$, because $T$ is normal); then by the above $\beta\left\lceil n \subset \varphi\left(v, \alpha\lceil(n+1)) \leq_{\text {lex }}\right.\right.$ $\varphi(u, \alpha \uparrow(n+2))$ and $\beta\left\lceil(n+1) \subset \varphi(u, \alpha \uparrow(n+2))\right.$ which imply $\varphi(v, \alpha \uparrow(n+1)) \leq_{\text {pw }}$ $\beta\lceil(n+1)$. Since $v \in S(\varphi(v, \alpha \uparrow(n+1)))$ and $S$ is normal we have also $v \in$ $S(\beta \uparrow(n+1))$, as needed.

Laver's proof [Lav71] of Fraïssé's conjecture implies that if in Definition 3.1 we allow only finitely many colors then the resulting quasi-order is a bqo, and hence very far from being $\Sigma_{1}^{1}$-complete (indeed it is well-founded and contains no infinite 
antichains, so that neither $\geq$ on $\mathbb{N}$ nor an infinite quasi-order with all elements incomparable are reducible to it). Therefore $\leq_{\mathbb{N} L O}$ is one of the simplest quasi-orders on $\mathbb{N}^{\mathrm{LO}}$ which is not bqo and Theorem 3.2 shows that it is indeed as complicated as it can be, namely $\boldsymbol{\Sigma}_{1}^{1}$-complete.

\section{$\S 4$. Dense order preserving functions.}

Definition 4.1. Suppose that $D$ and $E$ are countable dense linear orderings. A function $f: D \rightarrow E$ is dense order preserving if it is order preserving and satisfies the following condition:

$\forall q_{1}, q_{2} \in D \forall r_{1}, r_{2} \in E\left(f\left(q_{1}\right)<r_{1}<r_{2}<f\left(q_{2}\right) \longrightarrow \exists q \in D\left(r_{1}<f(q)<r_{2}\right)\right)$.

The above condition can be restated by saying that $f(D)$ is a dense subset of the least interval within $E$ containing its range (notice that this is stronger than requiring the range of $f$ to be a dense linear order).

Notice that the composition of two dense order preserving functions is dense order preserving.

Our interest in dense order preserving functions on the rationals is explained by the following fact.

Proposition 4.2. A function $f: \mathbb{Q} \rightarrow \mathbb{Q}$ is dense order preserving if and only if it is the restriction to $\mathbb{Q}$ of a continuous order preserving embedding $g: \mathbb{R} \rightarrow \mathbb{R}$ such that $g(\mathbb{Q}) \subseteq \mathbb{Q}$.

Proof. The if part is immediate. For the only if part, given $f: \mathbb{Q} \rightarrow \mathbb{Q}$ dense order preserving define $g$ by $g(x)=\sup \{f(q) \mid q \leq x\}$. Notice that $g$ extends $f$, is order preserving and hence one-to-one, and that the range of $g$ has no gaps and hence is an open interval in $\mathbb{R}$. Therefore $g$ is continuous.

Definition 4.3. Given a set $C$ and a countable dense linear order $D$ we use the set-theoretical notation $C^{D}$ to denote the set of all functions $c: D \rightarrow C$, i.e., of all colorings of $D$ with colors from $C$. We quasi-order $C^{D}$ by $c_{1} \leq_{\operatorname{dop}} c_{2}$ if and only if there exists $f: D \rightarrow D$ dense order preserving such that $c_{1}(q)=c_{2}(f(q))$ for all $q \in D$. If $J_{1}, J_{2} \subseteq D$ are intervals the definition of $c_{1}\left\lceil J_{1} \leq_{\text {dop }} c_{2}\left\lceil J_{2}\right.\right.$ is obvious.

THeOREM 4.4. If $|C| \geq 3$ there exists a sequence $\left(c_{n}\right)_{n \in \mathbb{N}}$ of elements of $C^{\mathbb{Q}}$ such that whenever $n<m$ we have $c_{n} \mid J \mathbb{Z}_{\mathrm{dop}} c_{m}$ for any unbounded interval $J \subseteq \mathbb{Q}$.

Proof. Fix $C_{0} \subseteq C$ such that $\left|C_{0}\right|=3$. For every $n$, let $C_{n+1}=\left\{a \subset C_{n} \mid\right.$ $|a|=2\}$, so that $\left|C_{n}\right|=3$ for every $n$.

For every $n$ we will consider the set $\mathbb{Q}^{n+1}$ of all sequences of $n+1$ rationals with lexicographic order. This linear order is order isomorphic to the usual order on $\mathbb{Q}$, and we fix an order isomorphism $\varphi_{n}: \mathbb{Q} \rightarrow \mathbb{Q}^{n+1}$ (obviously both $\varphi_{n}$ and its inverse $\varphi_{n}{ }^{-1}$ are dense order preserving). If $\bar{q} \in \mathbb{Q}^{i}$ with $i \leq n$ we let

$$
J_{\bar{q}}^{n}=\left\{\bar{r} \in \mathbb{Q}^{n+1} \mid \bar{q} \subset \bar{r}\right\} .
$$

Notice that $J_{\bar{q}}^{n}$ is an interval within $\mathbb{Q}^{n+1}$, and can be viewed as $\mathbb{Q}^{n+1-i}$. Moreover $J_{\bar{q}}^{n} \cap J_{\bar{p}}^{n}=\emptyset$ for every $\bar{p} \in \mathbb{Q}^{i}$ with $\bar{p} \neq \bar{q}$.

Fix $n$ : to define $c_{n}$ we inductively define $c_{n}^{i}: \mathbb{Q}^{n+1-i} \rightarrow C_{i}$, for $i=n, \ldots, 0$. We start by requiring that $c_{n}^{n}: \mathbb{Q} \rightarrow C_{n}$ is such that for every $a \in C_{n}$ the set 
$\left\{q \in \mathbb{Q} \mid c_{n}^{n}(q)=a\right\}$ is dense in $\mathbb{Q}$. If we have defined $c_{n}^{i+1}: \mathbb{Q}^{n-i} \rightarrow C_{i+1}$, we define $c_{n}^{i}$ so that the following two conditions are satisfied:

- $c_{n}^{i}(\bar{r}) \in c_{n}^{i+1}\left(\bar{r}\lceil n-i)\right.$ for every $\bar{r} \in \mathbb{Q}^{n+1-i}$;

- for any $\bar{q} \in \mathbb{Q}^{n-i}$ and $a \in c_{n}^{i+1}(\bar{q})$ the set $\left\{\bar{r} \in J_{\bar{q}}^{n-i} \mid c_{n}^{i}(\bar{r})=a\right\}$ is dense in $J_{\bar{q}}^{n-i}$.

Eventually we obtain $c_{n}^{0}: \mathbb{Q}^{n+1} \rightarrow C_{0}$ and let $c_{n}=c_{n}^{0} \circ \varphi_{n}$, so that indeed $c_{n} \in C^{\mathbb{Q}}$.

A straightforward induction shows that for every $i \leq n$ and every unbounded interval $J \subseteq \mathbb{Q}^{n+1-i}$ we have $c_{n}^{i} \leq_{\text {dop }} c_{n}^{i}\lceil J$. Thus for every unbounded interval $J \subseteq \mathbb{Q}$ we have $c_{n} \leq_{\text {dop }} c_{n}\left\lceil J\right.$. Therefore if $m>n$ to show that $c_{n}\left\lceil J \not_{\text {dop }} c_{m}\right.$ it suffices to show that $c_{n} \not \mathbb{Z}_{\text {dop }} c_{m}$.

Fix $n$ and $m$ with $n<m$ and suppose, towards a contradiction, that there exists $f: \mathbb{Q} \rightarrow \mathbb{Q}$ dense order preserving such that $c_{n}(q)=c_{m}(f(q))$ for all $q \in \mathbb{Q}$. For $i=0, \ldots, n$ we define $f_{i}: \mathbb{Q}^{n+1-i} \rightarrow \mathbb{Q}^{m+1-i}$ dense order preserving such that $c_{n}^{i}(\bar{q})=c_{m}^{i}\left(f_{i}(\bar{q})\right)$ for every $\bar{q} \in \mathbb{Q}^{n+1-i}$. We start by letting $f_{0}=\varphi_{m} \circ f \circ \varphi_{n}{ }^{-1}$ : it is straightforward to check that $f_{0}$ has the required properties.

Now suppose we have $f_{i}$ for some $i<n$. Given $\bar{q} \in \mathbb{Q}^{n-i}$ we define $f_{i+1}(\bar{q})$ to be the unique $\bar{r} \in \mathbb{Q}^{m-i}$ such that $f_{i}\left(J_{\bar{q}}^{n-i}\right) \subseteq J_{\bar{r}}^{m-i}$. To show that $f_{i+1}$ is well-defined we need to show that for every $\bar{q} \in \mathbb{Q}^{n-i}$ there exists such an $\bar{r}$ (which is obviously unique).

Fix $\bar{q} \in \mathbb{Q}^{n-i}$ and suppose that $\bar{r}, \bar{s} \in \mathbb{Q}^{m-i}$ are such that $f_{i}\left(J_{\bar{q}}^{n-i}\right)$ intersects both $J_{\bar{r}}^{m-i}$ and $J_{\bar{s}}^{m-i}$. Since $f_{i}$ is dense order preserving, there are intervals $J, J^{\prime} \subseteq J_{\bar{q}}^{n-i}$ such that $f_{i}(J) \subseteq J_{\bar{r}}^{m-i}$ and $f_{i}\left(J^{\prime}\right) \subseteq J_{\bar{s}}^{m-i}$. Using again the fact that $f_{i}$ is dense order preserving we may assume that $c_{m}^{i+1}(\bar{r}) \neq c_{m}^{i+1}(\bar{s})$ and hence at most one of these elements of $C_{i+1}$ coincides with $c_{n}^{i+1}(\bar{q})$. Suppose that $c_{n}^{i+1}(\bar{q}) \neq c_{m}^{i+1}(\bar{r})$ and let $a$ be the unique element of $C_{i}$ which belongs to $c_{n}^{i+1}(\bar{q})$ but not to $c_{m}^{i+1}(\bar{r})$. For all $\bar{t} \in J$ we have $c_{n}^{i}(\bar{t}) \neq a$; this contradicts the fact that $\left\{\bar{t} \in J_{\bar{q}}^{n-i} \mid c_{n}^{i}(\bar{t})=a\right\}$ is dense in $J_{\bar{q}}^{n-i}$.

To check that $f_{i+1}$ is order preserving it suffices to show that it is one-to-one: here the argument is similar to the one used to show that $f_{i+1}$ is well-defined, and we leave it to the reader.

To show that $f_{i+1}$ is dense suppose $f_{i+1}\left(\overline{q_{1}}\right)<_{\operatorname{lex}} \overline{r_{1}}<_{\operatorname{lex}} \overline{r_{2}}<_{\operatorname{lex}} f_{i+1}\left(\overline{q_{2}}\right)$. For $j=1,2$ pick $\bar{s}_{j} \in J_{\bar{q}_{j}}^{n-i}$ and $\overline{t_{j}} \in J_{\bar{r}_{j}}^{m-i}$, so that $f_{i}\left(\bar{s}_{1}\right)<_{\operatorname{lex}} \bar{t}_{1}<_{\operatorname{lex}} \overline{t_{2}}<_{\operatorname{lex}} f_{i}\left(\bar{s}_{2}\right)$. By induction hypothesis there exists $\bar{u} \in \mathbb{Q}^{n+1-i}$ with $\bar{t}_{1}<_{\operatorname{lex}} f_{i}(\bar{u})<_{\operatorname{lex}} \bar{t}_{2}$. Then $\overline{r_{1}} \leq_{\text {lex }} f_{i+1}(\bar{u} \mid n-i) \leq_{\operatorname{lex}} \overline{r_{2}}$.

Eventually we obtain $f_{n}: \mathbb{Q} \rightarrow \mathbb{Q}^{m+1-n}$ dense order preserving and such that $c_{n}^{n}(q)=c_{m}^{n}\left(f_{n}(q)\right)$ for every $q \in \mathbb{Q}$. Let $\bar{r} \in \mathbb{Q}^{m-n}$ be such that $J_{\bar{r}}^{m-n}$ intersects the range of $f_{n}$. Since $f_{n}$ is dense order preserving there exists an interval $J \subseteq \mathbb{Q}$ such that $f_{n}(J) \subseteq J_{\bar{r}}^{m-n}$. Since $c_{m}^{n+1}(\bar{r}) \in C_{n+1}$ there exists $a \in C_{n} \backslash c_{m}^{n+1}(\bar{r}): c_{m}^{n}(\bar{t}) \neq a$ for every $\bar{t} \in J_{\bar{r}}^{m-n}$ and hence $c_{n}^{n}(q) \neq a$ for every $q \in J$. This contradicts the fact that $\left\{q \in \mathbb{Q} \mid c_{n}^{n}(q)=a\right\}$ is dense in $\mathbb{Q}$.

The sequence $\left(c_{n}\right)_{n \in \mathbb{N}}$ constructed in the proof of Theorem 4.4 is actually descending (i.e., we have also $c_{m} \leq_{\text {dop }} c_{n}$ whenever $n<m$ ). Therefore $\leq_{\text {dop }}$ is not well-founded on $C^{\mathbb{Q}}$ when $|C| \geq 3$. However we will not need this fact and we leave its proof to the reader. 
There is another approach to the preceding result which was suggested to us by the referee. It shortcuts our explicit construction by using a sharpening of the classification result by Friedman and Stanley on countable linear orders [FS89]. A careful inspection of the construction by Friedman and Stanley leads to the following observation about the linear orders obtained there: whenever two of them are not isomorphic, each of them is not isomorphic to any interval of the other. In particular there exists an infinite sequence of linear orders each not isomorphic to any interval of the others.

Using this observation here is a sketch of the proof suggested by the referee: suppose $C=\{$ blue, red $\}$, and we wish to construct an infinite antichain in $C^{\mathbb{Q}}$ with respect to $\leq_{\text {dop. }}$. Given a countable linear order $\left(L,<_{L}\right)$ let $\left(L^{*},<_{L}^{*}\right)$ be the lexicographical product of $L$ with $2=\{0,1\}$ equipped with the natural ordering. (This means doubling each point of $L$.) Partition $\mathbb{Q}$ into disjoint open intervals ordered as $\left(L^{*},<_{L}^{*}\right)$ and color the points in the intervals corresponding to $L \times\{0\}$ blue and the points in the intervals corresponding to $L \times\{1\}$ red. Let $c_{L}: \mathbb{Q} \rightarrow C$ be the coloring obtained in this fashion. Now we can prove that if $c_{L} \leq_{\text {dop }} c_{L^{\prime}}$ for two linear orders $\left(L,<_{L}\right)$ and $\left(L^{\prime},<_{L^{\prime}}\right)$, we have that $\left(L,<_{L}\right)$ is isomorphic to an interval of $\left(L^{\prime},<_{L^{\prime}}\right)$. Thus the above observation about Friedman and Stanley's proof immediately yields an infinite antichain with respect to $\leq_{\text {dop }}$.

§5. Continuous embeddability between dendrites. We want to translate the combinatorial results of the previous sections into results about finitely branching dendrites. Our first goal is to mirror Theorem 4.4 on $\sqsubseteq^{c}$ restricted to finitely branching dendrites. To this end we need three finitely branching dendrites to play the role of the elements of $C_{0}$.

Definition 5.1. Let $D_{0}, D_{1}$ and $D_{2}$ be the finitely branching dendrites portrayed in figure 1 . For $i=0,1,2$ let $p_{i} \in D_{i}$ be the distinguished point marked in the same figure.
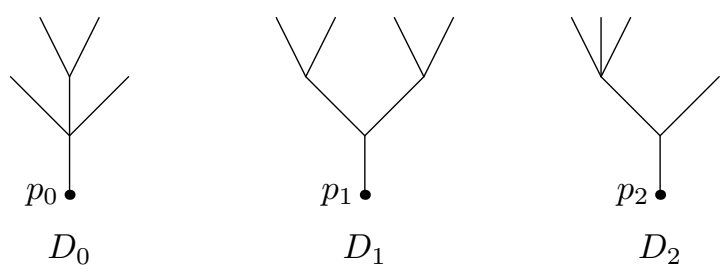

Figure 1. $D_{0}, D_{1}$, and $D_{2}$

$D_{0}$ and $D_{2}$ are actually homeomorphic, but the following incomparability holds.

Proposition 5.2. If $i \neq j$ there is no continuous embedding $g: D_{i} \rightarrow D_{j}$ such that $g\left(p_{i}\right)=p_{j}$.

Proof. This is immediate once noticed that for any such continuous embedding $g$ and any $x \in D_{i}$ we must have ord $\left(x, D_{i}\right) \leq \operatorname{ord}\left(g(x), D_{j}\right)$.

Definition 5.3. Let $\left(q_{k}\right)$ be a one-to-one enumeration of the rational numbers of the open interval $(0,1)$ and $\varphi: \mathbb{Q} \cap(0,1) \rightarrow \mathbb{Q}$ be a order isomorphism. 
For every $i=0,1,2$ and $k$ let $D_{i}^{k} \subseteq I^{2}$ be a homeomorphic copy of $D_{i}$ of diameter $<2^{-k}$, with the homeomorphism mapping $p_{i}$ to $p^{k}=\left(q_{k}, 0\right)$. We may assume that $D_{i}^{k} \cap(I \times\{0\})=\left\{p^{k}\right\}$ and that $D_{i}^{k} \cap D_{j}^{k^{\prime}}=\emptyset$ whenever $k \neq k^{\prime}$.

For every $n$ let $c_{n}$ be the function of Theorem 4.4 and define

$$
X_{n}=(I \times\{0\}) \cup \bigcup_{k \in \mathbb{N}} D_{c_{n}\left(\varphi\left(q_{k}\right)\right)}^{k} .
$$

It is clear that $X_{n}$ is a finitely branching dendrite. Theorem 4.4 translates to the following fact.

Lemma 5.4. If $n<m, X_{n} \cap([x, 1] \times I) \unrhd^{c} X_{m}$ for any $x \in[0,1)$.

Proof. Suppose that $x \in[0,1)$ and that $g: X_{n} \cap([x, 1] \times I) \rightarrow X_{m}$ is a continuous embedding. Then $g$ maps branching points into branching points, and must map the $\operatorname{arc}[x, 1] \times\{0\}$, which has a dense subset of branching points, into $I \times\{0\}$, the only arc contained in $X_{m}$ with this property. Moreover the rational points of $(x, 1) \times\{0\}$ are mapped into the rational points of $I \times\{0\}$ and Proposition 5.2 implies that $g$ maps a point with $D_{i}$ attached to a point with the same $D_{i}$ attached.

Hence, restricting ourselves to the first coordinate and using $\varphi$ to transfer everything into $\mathbb{Q}$, we obtain a function $f: J \rightarrow \mathbb{Q}$ such that $c_{n}(q)=c_{m}(f(q))$ for every $q \in J$, where $J$ is some final segment of $\mathbb{Q}$. Since $f$ is the restriction of a continuous embedding of the reals to $\mathbb{Q}$, Property 4.2 implies that if $f$ is increasing then it is dense order preserving, contradicting Theorem 4.4. If $f$ is decreasing we can observe that Theorem 4.4 holds also if we allow functions which are order reversing, since the $c_{n}$ 's have been defined in a symmetric way with respect to the order.

We now build an antichain of finitely branching dendrites with respect to $\coprod^{c}$.

Definition 5.5. For any $k$ and $i \in\{0,1\}$ let $A_{k}^{i}$ be an arc of length $<2^{-k}$ with $(i, 0)$ as one of its end points. We may assume that $A_{k}^{i} \cap X_{n}=\{(i, 0)\}$ for every $n$ and that $A_{k}^{i} \cap A_{k^{\prime}}^{i}=\{(i, 0)\}$ whenever $k \neq k^{\prime}$, while $A_{k}^{0} \cap A_{k^{\prime}}^{1}=\emptyset$ for any $k, k^{\prime}$. Let

$$
Y_{n}=X_{n} \cup \bigcup_{k<n+4} A_{k}^{0} \cup \bigcup_{k<n+5} A_{k}^{1} .
$$

The base of $Y_{n}$ is the $\operatorname{arc} I \times\{0\}$.

It is clear that $Y_{n}$ is a finitely branching dendrite and that $(0,0)$ and $(1,0)$ have order respectively $n+5$ and $n+6$ in $Y_{n}$. Moreover $n+6$ is the maximal order of a point in $Y_{n}$, since all other points have order at most 4 .

Lemma 5.6. If $n \neq m, Y_{n} \cap([x, 1] \times I) \unrhd^{c} Y_{m}$ for any $x \in[0,1)$.

Proof. If $n<m$ this follows immediately from Lemma 5.4, since it is clear that $X_{n} \cap([x, 1] \times I) \square^{c} Y_{m}$ for any $x \in[0,1)$.

If $n>m$ observe that $Y_{n} \cap([x, 1] \times I)$ contains a point of order $n+6$, while the point of maximal order in $Y_{m}$ has order $m+6$.

LEMMA 5.7. Every homeomorphism of $Y_{n}$ into itself maps is the identity on $(0,0)$ and $(1,0)$.

Proof. This is immediate taking into account the order of the points.

The proof of the Main Theorem uses the $Y_{n}$ 's to mimic the colors of section 3. 
Proof of Main Theorem. By Theorem 3.2 it suffices to Borel reduce $\leq_{\mathbb{N}^{\text {Lo }}}$ to $\coprod^{c}$ on finitely branching dendrites.

Let $Q^{\prime} \subset I$ be discrete in the relative topology and order isomorphic to $\mathbb{Q}$ (e.g., embed $\mathbb{Q} \times 3$ with $\leq_{\text {lex }}$ into $\mathbb{Q}$ and let $Q^{\prime}$ be the image of $\left.\mathbb{Q} \times\{1\}\right)$. For every $q \in Q^{\prime}$ let $\varepsilon_{q}>0$ be such that $0<q-\varepsilon_{q}, q+\varepsilon_{q}<1$ and $\left(q-\varepsilon_{q}, q+2 \varepsilon_{q}\right) \cap Q^{\prime}=\{q\}$. Let $I_{q}=\left[q, q+\varepsilon_{q}\right]$.

If $\mathfrak{A}=\left(L_{A}, f_{A}\right) \in \mathbb{N}^{\mathrm{LO}}$ we can define in a continuous way a function $g_{A}: \mathbb{N} \rightarrow Q^{\prime}$ such that $a L_{A} a^{\prime}$ if and only if $g_{A}(a)<g_{A}\left(a^{\prime}\right)$.

Let $Z_{\mathfrak{A}}$ be the union of $I \times\{0\}$ and of a homeomorphic copy of $Y_{f_{A}(a)}$ contained in $I_{g_{A}(a)} \times\left[0,2^{-a}\right]$ with base $I_{g_{A}(a)} \times\{0\}$ and $\left(g_{A}(a), 0\right)$ corresponding to $(0,0)$, for each $a \in \mathbb{N}$. Notice that $Z_{\mathfrak{A}}$ is a finitely branching dendrite.

The function $\mathfrak{A} \mapsto Z_{\mathfrak{A}}$ is continuous and we need to show that $\mathfrak{A} \leq_{\mathbb{N} \text { LO }} \mathfrak{B}$ is equivalent to $Z_{\mathfrak{A}} \sqsubseteq^{c} Z_{\mathfrak{B}}$ for every $\mathfrak{A}, \mathfrak{B} \in \mathbb{N}^{\mathrm{LO}}$.

If $\mathfrak{A} \leq_{\mathbb{N} L O} \mathfrak{B}$ with $\mathfrak{A}=\left(L_{A}, f_{A}\right)$ and $\mathfrak{B}=\left(L_{B}, f_{B}\right)$ let $\psi: \mathbb{N} \rightarrow \mathbb{N}$ be a witness. To define a continuous embedding $F: Z_{\mathfrak{A}} \rightarrow Z_{\mathfrak{B}}$ notice that since $f_{A}(a)=f_{B}(\psi(a))$ for every $a \in \mathbb{N}$ there is a homeomorphism between the homeomorphic copy of $Y_{f_{A}(a)}$ contained in $I_{g_{A}(a)} \times\left[0,2^{-a}\right]$ and the homeomorphic copy of $Y_{f_{B}(\psi(a))}$ contained in $I_{g_{B}(\psi(a))} \times\left[0,2^{-\psi(a)}\right]$. Let $F$ contain the union of all these automorphisms (which have disjoint domains and disjoint ranges). By Lemma 5.7, $F\left(g_{A}(a), 0\right)=\left(g_{B}(\psi(a)), 0\right)$ for every $a \in \mathbb{N}$. So far $F$ has been defined on $Z_{\mathfrak{A}}$ except on some subarcs of $I \times\{0\}$ and possibly in $(0,0)$ and $(1,0)$. The complement in $Z_{\mathfrak{B}}$ of the range of the function $F$ defined so far contains (by the choice of $\varepsilon_{q}$ ) corresponding subarcs of $I \times\{0\}$. Therefore $F$ can be extended to the whole of $Z_{\mathfrak{A}}$.

Now suppose $Z_{\mathfrak{A}} \sqsubseteq^{c} Z_{\mathfrak{B}}$ and let $F$ be the continuous embedding. It is immediate that $F$ maps $I \times\{0\}$ into itself, so that $F(x, 0)=(h(x), 0)$ for some continuous embedding $h: I \rightarrow I$.

We claim that $h$ is order preserving. If this were not the case we should have $h(x)>h\left(x^{\prime}\right)$ whenever $x<x^{\prime}$. Since $\left(g_{A}(a)+\varepsilon_{g_{A}(a)}, 0\right)$ is the limit from the left of branching points, while $\left(g_{B}(b)+\varepsilon_{g_{B}(b)}, 0\right)$ is not the limit from the right of branching points, we have $h\left(g_{A}(a)+\varepsilon_{g_{A}(a)}\right) \neq g_{B}(b)+\varepsilon_{g_{B}(b)}$ for any $a, b \in \mathbb{N}$. Hence for any $a \in \mathbb{N}$ we must have $h\left(g_{A}(a)+\varepsilon_{g_{A}(a)}\right)=g_{B}(b)$ for some $b \in \mathbb{N}$. This implies (again by looking at the order of the branching points) that $f_{A}(a)<f_{B}(b)$. Since $F$ continuously embeds a final piece of $X_{f_{A}(a)}$ into $X_{f_{B}(b)}$ we contradict Lemma 5.4 and the proof of the claim is complete.

Thus $h$ is order preserving and if $a \in \mathbb{N}$ Lemmas 5.6 and 5.7 imply that $h\left(g_{A}(a)+\right.$ $\left.\varepsilon_{g_{A}(a)}\right)=g_{B}(b)+\varepsilon_{g_{A}(b)}$ for some $b \in \mathbb{N}$ such that $f_{A}(a)=f_{B}(b)$. Let $\psi(a)=b$. The function $\psi$ shows that $\mathfrak{A} \leq_{\mathbb{N} L O} \mathfrak{B}$.

Camerlo, Darji and Marcone in [CDM02] studied homeomorphism on the class of dendrites which have all branching points contained in an arc. This class arises naturally from the study of the likeness relation among dendrites (see [CDM02]) and it is natural to ask the following question.

QUESTION 5.8. Is $\sqsubseteq^{c}$ restricted to dendrites which have all branching points contained in an arc $\Sigma_{1}^{1}$-complete? 


\section{REFERENCES}

[BK96] Howard Becker and AleXander S. Kechris, The descriptive set theory of Polish group actions, Cambridge University Press, Cambridge, 1996.

[CDM02] Riccardo Camerlo, Udayan B. Darji, and Alberto Marcone, Classification problems in continuum theory, preprint, 2002.

[FS89] Harvey Friedman and Lee Stanley, A Borel reducibility theory for classes of countable structures, this JournaL, vol. 54 (1989), no. 3, pp. 894-914.

[Hjo00] GREG HJORTH, Classification and orbit equivalence relations, American Mathematical Society, Providence, RI, 2000

[Kec95] AleXANDER S. KeChris, Classical descriptive set theory, Graduate Texts in Mathematics, no. 156, Springer-Verlag, New York, 1995.

[Lav71] Richard LaVER, On Fraïssé's order type conjecture, Annals of Mathematics (2), vol. 93 (1971), pp. 89-111.

[LR02] Alain Louveau and Christian Rosendal, Complete analytic equivalence relations, preprint, 2002

[Nad92] SAm B. NAdLeR, JR., Continuum theory, Marcel Dekker Inc., New York, 1992.

DIPARTIMENTO DI MATEMATICA E INFORMATICA

UNIVERSITÀ DI UDINE

VIA DELLE SCIENZE 208

33100 UDINE, ITALY

E-mail: marcone@dimi.uniud.it

ÉQUIPE D'ANALYSE FONCTIONNELLE

UNIVERSITÉ PARIS 6

TOUR 46-0, BOÎTE 186; 4, PLACE JUSSIEU

75252 PARIS. FRANCE

E-mail: rosendal@ccr.jussieu.fr 